\title{
Pneumococcal serotypes in children and relationship with clinical presentation and antimicrobial susceptibility in the PCV13 era
}

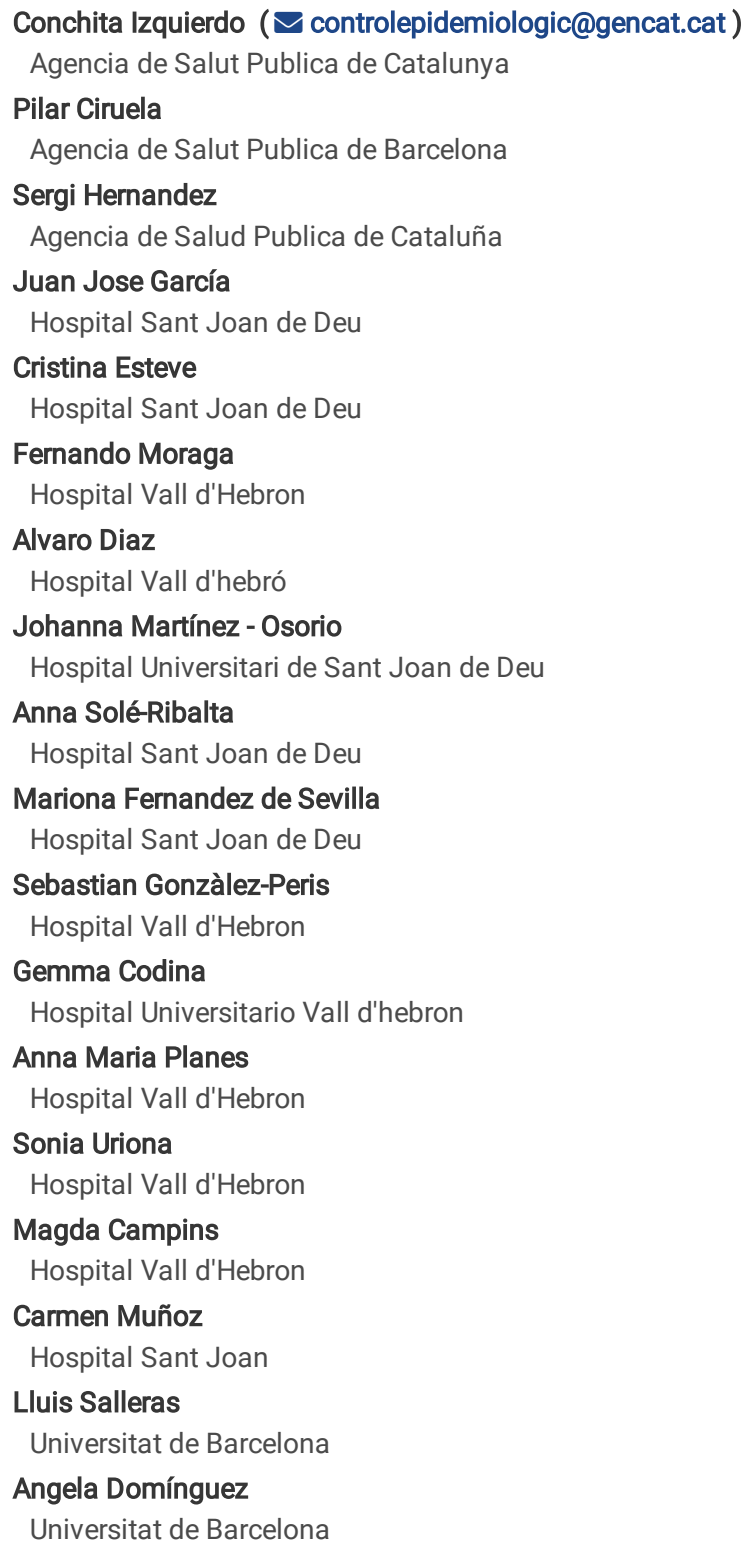

\section{Research article}

Keywords: Streptococcus pneumoniae; serotype distribution; PCV13 vaccine, Invasive pneumococcal disease; antimicrobial susceptibility

Posted Date: August 20th, 2019

DOI: https://doi.org/10.21203/rs.2.13143/v1

License: () (1) This work is licensed under a Creative Commons Attribution 4.0 International License. Read Full License 


\section{Abstract}

Background: The aim of this study was to analyse the serotypes causing invasive pneumococcal disease (IPD) according to the clinical presentation, and antimicrobial susceptibility in children aged $\leq 17$ years before the introduction of the 13-valent pneumococcal conjugate vaccine (PCV13) in the official paediatric schedule. Methods: We conducted a prospective study in children $\leq 17$ years with IPD attended in three Catalan hospitals between January 2012 and June 2016. IPD cases were diagnosed by culture or RT-PCR. Demographic, microbiological, and clinical data were analysed. Associations were assessed using the odds ratio (OR) and 95\% confidence intervals $(\mathrm{Cl})$. Results: Of the 253 cases included, $34.4 \%$ were aged $<2$ years, $38.7 \% 2-4$ years and $26.9 \% 5-17$ years. Over $64 \%$ of cases were PCV13 serotypes. Cases with PCV13 serotypes were associated with overall pneumonia (OR:7.47[4.0-13.96]) and complicated pneumonia (OR:7.2[4.04-12.75]), overall and in each age group $(p<0.05)$. Serotypes 3 and 1 were associated with complicated pneumonia $(p<0.05)$. Non-PCV13 serotypes were associated with meningitis (OR:7.32[2.33-22. 99]) and occult bacteremia (OR:3.6[1.56-8.76]). Serotype 19A was more frequent in children aged $<2$ years and serotype 3 and 1 in those aged 2-4 years and $>4$ years, respectively. Forty-four cases (36.1\%) were non-susceptible to penicillin and $16.4 \%$ were also non-susceptible to cefotaxime. There were no significant differences between PCV13 and non-PCV13 cases with nonsusceptible penicillin strains (36.1\% and $36.0 \%$, respectively), while PCV13 cases showed more frequently non-susceptible cefotaxime strains (23.6\%; $p=0.010)$. Serotypes $19 A$ and 14 were associated with non-susceptibility to both penicillin and cefotaxime strains ( $p=0.003$ and $p<0.001$, respectively) and serotype 19A with resistant to penicillin ( $p=0.002)$ Conclusions: PCV13 serotypes were the most frequent serotypes in children aged $\leq 17 y e a r s$, mainly serotype 3, 1 and 19A. Non-PCV13 serotypes were associated with meningitis and occult bacteraemia while PCV13 serotypes with pneumonia. PCV13 and non-PCV13 cases presented high frequency of non-susceptibility to penicillin. Serotypes 19A, and 14 were associated with non-susceptibility to both penicillin and cefotaxime, and serotype19A with penicillin resistance.

\section{Background}

Streptococcus pneumoniae infections are associated with substantial morbidity and mortality worldwide. The World Health Organization estimated that approximately $335,000(240,000-460,000)$ deaths were due to S. pneumoniae in children aged $<5$ years in 2015 [1].

The epidemiology of invasive pneumococcal disease (IPD) has changed after the introduction of pneumococcal conjugate vaccines [2].In countries where the 7-valent pneumococcal conjugate vaccine (PCV7) (with serotypes 4, 6B, 9V, 14, 18C, 19F and 23F) was introduced, the global IPD incidence decreased but the non-PCV7 serotypes emerged [2-5]. Later, higher-valent vaccines [10-valent pneumococcal conjugate vaccine (PCV10), that includes PCV7 serotypes plus serotypes 1, 5 and 7F, and 13-valent pneumococcal conjugate vaccine (PCV13) that includes PCV10 serotypes plus 3, 6A and 19A] [6] were introduced and a significant decline in some of additional serotypes was observed [7].

In Catalonia, Spain, PCV7 was available in 2001, but only children with risk factors were vaccinated for free [8,9]. An estimated vaccine coverage of around $50 \%$ was reached [9].Between 2005 and 2007, a change in IPD serotypes was found in children aged <2 years, compared with the pre-vaccine period, with a decrease in PCV7 serotypes and an increase in non-PCV7 serotypes [10]. PCV13 replaced PCV7 in 2010 and finally, in July 2016, was included in the paediatric official calendar [11]. PCV13 could cover up to 70\% of IPD cases in our reference area [12].

Studies worldwide have found that the changing distribution of pneumococcal serotypes has led to changes in the rates of $S$. pneumoniae resistance to antibiotics $[13,14]$.

The aim of this study was to analyse the distribution of $S$. pneumoniae serotypes according to the clinical presentation of IPD and antimicrobial susceptibility in children aged $<18$ years in a community with intermediate PCV13 coverage before the introduction of this vaccine in the official paediatric schedule.

\section{Methods}

\subsection{Study design}

We conducted a prospective observational study between January 2012 and June 2016 in children aged <18 years with IPD attended in three paediatric hospitals: Hospital Vall d'Hebron of Barcelona, Hospital Sant Joan de Déu of Esplugues, Barcelona and Hospital de Nens of Barcelona. The estimated reference population aged $<18$ years of these hospitals was 442,761 , representing $31.9 \%$ of this age group in Catalonia.

IPD was diagnosed through the isolation of $S$. pneumoniae by culture or detection of bacterial DNA by real-time polymerase chain reaction (RT-PCR) in any normally sterile site [15].

\subsection{Data collected}

Epidemiological and clinical characteristics, including age, gender, underlying medical condition, clinical presentation, intensive care unit (ICU) admission, clinical outcome and PCV13 vaccination status were collected. The method of diagnosis, serotype and antimicrobial susceptibility were also analysed.

A subject was considered vaccinated if they had received the last dose of PCV13 $\geq 15$ days before symptom onset.

\subsection{Serotype identification and antimicrobial susceptibility}

Strains isolated by culture were serotyped using the Quellung reaction or dot-blot at the National Centre of Microbiology (Majadahonda, Spain) [16]. When the diagnosis was made only by RT-PCR, serotypes were identified at the Molecular Microbiology Department, Hospital Sant Joan de Déu in accordance with 
previously-validated methods [15].The procedure allows the identification of 40 serotypes by a RT-PCR threshold cycle $\leq 30$.

Serotypes were categorized into PCV13 serotypes (4, 6B, 9V, 14, 18C, 19F, 23F, 1, 5, 7F, 3, 6A and 19A) and non-PCV13 serotypes (all other serotypes). Serotypes identified by RT-PCR as group level (6A/6C, 9V/A, 19FBC, and 7FA) were defined as PCV13 serotypes 6A, 9V, 19F and 7F, respectively.

Susceptibility to penicillin and cefotaxime were determined by the microdilution method according to the Clinical and Laboratory Standards Institute (CLSI) criteria. Current EUCAST breakpoints were used to interpret susceptibility [17]. A minimum inhibitory concentration (MIC) study was carried out at the National Microbiology Center. The penicillin breakpoints used were: MIC $>0.06 \mathrm{mg} / \mathrm{L}$, non-susceptible, and MIC $>2 \mathrm{mg} / \mathrm{L}$, resistant. For cefotaxime breakpoint was: $\mathrm{MIC}>0.5 \mathrm{mg} / \mathrm{L}$, non-susceptible.

\subsection{Statistical analysis}

Proportions were compared using the $\chi^{2}$ test or Fisher's exact test, as appropriate. All statistical tests were two tailed and statistical significance was established as $p<0.05$. If the number of cases was zero, this was corrected by adding 0.5 , and $p$-values were calculated according to Sheskin's method [18]

Associations between variables were assessed using the odds ratio (OR) and 95\% confidence intervals (Cl).

The analyses were conducted using the Statistical Package for Social Sciences (SPSS 19.0 for Windows) and EPIDAT (program for the epidemiological analysis of tabulated data; version 3.1).

\subsection{Data confidentiality and ethical aspects}

Informed consent was obtained from all individual participants included in the study. The study complies with the principles of the Declaration of Helsinki and the legal structure with respect to international human rights and biomedicine and protection of personal data laws. The Ethics Committee of Hospital Sant Joan de Déu approved the study.

\section{Results}

\subsection{Baseline characteristics}

During the study period, 263 cases of IPD were diagnosed in children aged $<18$ years. Serotyping was not possible in 10 cases (3.8\%), which were excluded. Of the 253 cases included, 151 (59.7\%) were male; 87 (34.4\%) were in children aged <2 years, 98 (38. $7 \%$ ) in those aged $2-4$ years, and 68 (26.9\%) in those aged $5-17$ years (Table 1$)$.

There was a seasonal variation, with $72 \%$ of cases detected during the cool months (October to March; $p<0.001)$.

Eighty-three patients (32.8\%) had received $\geq 1$ dose of PCV13, while 129 (51.0\%) had received no dose of any conjugate vaccine.

Fifteen cases (6.0\%) had a chronic disease, including congenital immunodeficiency (2 cases), congenital heart disease (3 cases), chronic pulmonary disease ( 1 case), chronic kidney disease ( 1 case), and cerebrospinal fluid leak (1 case), or had received immunosuppressive therapy in the previous 6 months ( 7 cases).

The most frequent clinical presentation was pneumonia (187 cases; $73.9 \%$ ), of which 150 cases (80. 2\%) were complicated pneumonia. It was followed by occult bacteraemia ( 25 cases; $9.9 \%$ ) and meningitis (18 cases; $7.1 \%)$. The presentation in the remaining 23 cases (9.1\%) was septic shock (7 cases; $2.8 \%$ ), mastoiditis (7 cases; $2.8 \%$ ), osteoarticular infection ( 6 cases; $2.3 \%$ ), orbital cellulitis ( 2 cases; $0.8 \%$ ) and pancreatitis ( 1 case; $0.4 \%$ ). Figure 1 shows the distribution of clinical manifestations by age group. Complicated pneumonia was the most frequent clinical presentation in all age groups.

Forty-nine cases (19.4\%) were admitted to the ICU: 7 of 7 (100\%) with septic shock, 15 of 18 (83.3\%) with meningitis, 23 of 150 (15.3\%) with complicated pneumonia, 1 of 7 (14.3\%) with mastoiditis, 2 of 37 (5.4\%) with non-complicated pneumonia and 1 of 25 (4\%) with occult bacteraemia (in this case ICU admission was caused by convulsions). Of the 49 ICU cases, $55.1 \%$ were caused by PCV13 serotypes, and $14.3 \%$ had an underlying disease.

Two patients died: an 18-month-old child with serotype-35F meningitis who had congenital immunodeficiency, and a 23-month-old child with septic shock caused by a non-vaccine serotype which was not identified.

\subsection{Serotypes and clinical presentation}

One hundred and sixty-three (64.4\%) cases were due to PCV13 serotypes (Figure 2); It was $41.5 \%$ in patients who had received $\geq 1$ dose of PCV13 (34/82 cases) and $75.2 \%$ in patients who had not received any conjugated vaccine $(97 / 129 ; p<0.001)$.

The most frequent serotypes were: 3 (20.9\%), 1 (19.0\%), 19A (6.7\%) and 14 (5.9\%) all included in PCV13.

Overall and complicated pneumonia were mainly caused by PCV13 serotypes (143/187 cases, 76.4\% and 123/150 cases, 82.0\%, respectively). PCV13 serotypes caused overall pneumonia in $87.7 \%$ of cases and non-PCV13 serotypes in 48.9\% (OR: 7.47 [4.0-13.96]). Similarly, PCV13 serotypes led to complicated pneumonia in 75.5\% of cases and non-PCV13 serotypes in 30\% (OR: 7.2 [4.04-12.75]) (Table 2). 
Serotype 3 caused overall pneumonia in $92.5 \%$ of cases, especially complicated pneumonia (83.0\%), and was associated with both clinical presentations (OR:5.5 [1.9-15.92] and, OR:4.3 [2.01-9.35], respectively). Serotype 1 was also associated with overall pneumonia (OR: 21.82 [2.95-161.63]) and complicated pneumonia (OR: 5.16 [2.21-12.04]).

Meningitis was mainly caused by non-PCV13 serotypes (14/18, $77.8 \%)$. Although there was no predominant serotype, non-PCV13 serotypes caused meningitis in $15.6 \%$ of cases and PCV13 serotypes in 2.5\% (OR: 7.32 [2.33-22.99]).

Occult bacteraemia was also caused mainly by non-PCV13 serotypes (16/25, 64\%). Non-PCV13 serotypes caused occult bacteraemia in $17.8 \%$ of cases and PCV13 serotypes in 5.5\% (OR: 3.6 [1.56-8.76]). Some non-PCV13 serotypes were associated with occult bacteraemia namely 12F (OR: 19.73 [1.72226.12]) and 10A (OR: 10.22 [1.94-53.74]). Also serotype 18C (PCV13 serotype), showed a slight association (OR: 48.61 [2.26-1043.02]).

Other clinical presentations were caused mainly by non-PCV13 serotypes (16/23; 69.6\%). Non-PCV13 serotypes caused these presentations in $17.8 \%$ of cases and PCV13 in 4.3\% (OR: 4.81 [1.90-12.22]).

\subsection{Serotypes and clinical presentation according to age group}

PCV13 serotypes were most frequent in the $5-17$ years age group (77.9\%; OR: 2.41 [1.27-4.59]) and non-PCV13 in $<5$ years, mainly in the $<2$ years age group (55.2\% OR: 3.63 [2.10-6.29]. Serotype 19A was more frequent in the <2 years age group (58.8\%; OR: 2.95 [1.08-8.05]), serotype 3 in the $2-4$ years age group (58.5\%; OR: 2.79 [1.50-5.20]) and serotype 1 in the 5-17 years age group (60.4\%; OR: 6.50 [3.31 -12.77]) (Figure 2).

Table 3 and figure 3 show the serotype distribution by age groups and the association to clinical presentation.

In children aged <2 years, PCV13 serotypes were associated with overall pneumonia (OR: 5.29 [2.08-13.41]) and complicated pneumonia (OR: 4.84 [1.9112.22]). Serotype 3 caused overall pneumonia in $91.7 \%$ of cases (OR: 12.57 [1.54-102.33]) and complicated pneumonia in 83.3\% (OR: 10.62 [2.16-52.3]). Serotype 19A was associated with complicated pneumonia (OR: 4.32 [1.03-18.07]) in 70\% of cases.

In the 2-4 years age group, PCV13 serotypes were associated with overall pneumonia (OR: 5.42 [1.70-17.23]) and complicated pneumonia (OR: 6.92 [2.6118.36]), while non-PCV13 serotypes were associated with meningitis (OR: 27.38 [1.42-527.7]). Serotype 3 was associated with complicated pneumonia (OR: 6.7 [1.85-24.24]) and serotype 9V with occult bacteraemia (OR: 102.7 [4.27-2472.3]).

In the 5-17 years age group, PCV13 serotypes were associated with overall pneumonia (OR: 8.17 [1.91-34.86]) and complicated pneumonia (OR: 6.05 [1.81-20.18]), whereas non-PCV13 serotypes were associated with other clinical forms [OR: 9.3 [1.51-57.12]]. Serotype 1 was associated with complicated pneumonia (OR: 10.43 [2.17-50.12])

\subsection{Serotypes and method of diagnosis}

The diagnosis was made by culture in 62 cases (24.5\%), culture and RT-PCR in 69 (27.3\%) and by RT-PCR in 122 (48.2\%).

PCV13 and non-PCV13 serotypes showed no significant difference in the percentage of cases diagnosed exclusively by PCR (52.1\% versus $41.1 \%$ ).

There were significant differences between serotypes in the method of diagnosis: $86.8 \%$ of cases due to serotype 3 were diagnosed only by PCR, versus $38 \%$ in the other serotypes $(\mathrm{p}<0.001)$. By contrast, other serotypes, such as serotype 14 and $24 \mathrm{~F}$ were diagnosed less frequently by PCR $(13.3 \%, 2 / 15$ and $0 \%$, $0 / 6)$ than the other serotypes ( $p=0.005$ and $p=0.030$, respectively).

\subsection{Non-susceptible antibiotics by serotypes}

Antimicrobial susceptibility was studied in 122/131 (93.1\%) strains isolated. Forty-four cases (36.1\%) were non-susceptible to penicillin, and 4 cases (3.3\%) were penicillin resistant. Twenty cases (16.4\%) were also non-susceptible to cefotaxime (Table 4).

There were no significant differences in the percentage of non-susceptible penicillin strains between PCV13 serotypes (36.1\%) and non-PCV13 serotypes (36.0\%). PCV13 serotypes were associated with strains not susceptible to cefotaxime $(p=0.010)$ and with isolates not susceptible to both penicillin and cefotaxime $(p=0.010)$.

Serotype 19A showed a penicillin MIC $>0.06 \mathrm{mg} / \mathrm{L}$ in $90.9 \%$ of cases studied, $(\mathrm{p}<0.001)$ and $\mathrm{MIC}>2 \mathrm{mg} / \mathrm{L}$ in $27.3 \%$ ( $p=0.002)$. In addition, $54.5 \%$ of serotype 19A strains were non-susceptible to cefotaxime $(p=0.003)$ and also non-susceptible to penicillin $(p=0.003)$.

All serotype 14 strains (100\%) showed a penicillin MIC $>0.06 \mathrm{mg} / \mathrm{L}(\mathrm{p}<0.001)$, and $81.8 \%$ of cases were non-susceptible to both cefotaxime ( $\mathrm{p}<0.001)$ and penicillin $(p<0.001)$.

Serotype $11 \mathrm{~A}$ showed non-susceptibility to cefotaxime in $60 \%$ of strains compared with $14.5 \%$ for other serotypes $(p=0.031)$. These strains also were nonsusceptible to penicillin $(p=0.031)$.

Other serotypes showed significant differences in antimicrobial susceptibility, namely $24 \mathrm{~F}$ which showed penicillin $\mathrm{MIC}>0.06 \mathrm{mg} / \mathrm{L}$ in $83.3 \%(p=0.023)$, and $23 B$ in $100 \%(p=0.045)$.

\section{Discussion}


PCV13 serotypes caused $64.4 \%$ of IPD in children aged $<18$ years during the study period, and the most frequent serotypes were $3,1,19 \mathrm{~A}$ and 14 . Other studies in Catalonia have shown a higher frequency of PCV13 serotypes despite the decrease in the incidence in all age groups in this period [19].However, other authors have found a higher percentage of non-PCV13 serotypes. Makwana et al observed that 77.5\% of cases of IPD in children aged $<5$ years in England and Wales between 2010 and 2016 were caused by non-PCV13 serotypes [20]. A study conducted in Madrid (Spain) between 2012 and 2015, found that this percentage was $68.2 \%$ in children aged $<15$ years [21], a figure a little higher than the $55.2 \%$ described by Janoir et al [14] in France in $2011-2012$ in the same age group. The differences between these results might be explained, at least in part, by a lower PCV13 coverage in Catalan children than in other areas because, during the study period, PCV13 was not included in the Catalan immunization schedule.

In relation with the distribution of the main serotypes, Ceyhan et al [22] found that, in Turkish children in the same age group than us, serotype 19F was the most frequent, followed by 14, 3 and 6B (in equal amounts), while Camilli et al [23], in Italian children aged $<5$ years in 2012-2014, found that serotypes 1 , $19 \mathrm{~A}$ and 14 were among the four most frequent serotypes, together with 24F. The diagnostic techniques used in these studies may be one of the main explanatory factors for these differences [24]. In our study, serotype 3 was the most frequent and the most frequently diagnosed only by PCR (87\% of cases due to serotype 3). When PCR is not available for the diagnosis, another distribution of serotypes in the same age groups may be found, due to under detection.

Serotype 19A was associated with age <2 years, serotype 3 with the $2-4$ years age group and serotype 1 with the 5-17 years age group. Other studies have found similar results, with $100 \%$ of cases due to serotype $19 \mathrm{~A}$ in children aged $<2$ years, $63.6 \%$ of cases due to serotype 3 in the $2-4$ years age group, and $80 \%$ of cases due to serotype 1 in children aged $>4$ years [21].

PCV13 serotypes were associated with overall pneumonia and complicated pneumonia in children < 18 years, mainly serotype $19 \mathrm{~A}$ in children aged < 2 years, serotype 3 in those aged $<5$ years and serotype 1 in the 5-17 years age group. In a Polish study conducted between 2011 and 2013 in all age groups, it was observed that PCV13 serotypes caused $78.4 \%$ of cases of pneumonia, and serotype 3 and serotype 1 were associated to pneumonia as our study [25].

In our study, non-PCV13 serotypes were associated with occult bacteraemia, especially $10 \mathrm{~A}$ and $12 \mathrm{~F}$, as well as meningitis, which showed this association only to in the 2-4 years age group. Similarly, other authors have reported that $90 \%$ of meningitis cases and $64 \%$ of occult bacteraemia cases during $2011-$ 2012 were produced by non-PCV13 serotypes [26].

The percentage of strains not susceptible to penicillin (36.1\%) or to cefotaxime (16.4\%) was higher than that described by other authors [23,25]. Serotypes $19 \mathrm{~A}$ and 14 were associated with strains not susceptible to penicillin, nor to cefotaxime, whereas serotype 23B and 24F were not susceptible to penicillin. Other authors have also described non-susceptibility to penicillin in serotypes 19A and 14 [23,25], in serotype 24F [14,23] or in serotype 23B [27]. Skoczyńska et al [25] found that not susceptibility to penicillin besides was associated with the vaccine-serotypes 19F, 6B and 9V, which in our study could not be demonstrated, perhaps due to the small number of strains studied. They found association of not susceptibility to cefotaxime in serotype $19 \mathrm{~A}$ and 14 as in our study. The non-susceptibility to antibiotics of non-PCV13 serotypes previously commented is a worrisome fact and should be monitored.

Among the strengths of the study is the high percentage of cases serotyped, since serotyping was not possible in only $3.8 \%$ of cases. In addition, the same diagnostic techniques (culture and PCR) were used uniformly in all cases, improving both the diagnostic sensitivity and the range of serotypes identified, which permitted a more precise estimate of the burden of IPD.

There were some limitations. First, the population studied corresponds to three paediatric hospitals, although these hospitals serve $32 \%$ of the population aged $<18$ years requiring hospitalization in Catalonia. In the subgroup analyses, the small number of cases observed in some situations may explain that the confidence intervals of the associations were very wide. Finally, antibiotic sensitivity could only be studied in $48 \%$ of cases serotyped, since a large part of the cases were diagnosed by PCR.

\section{Conclusions}

PCV13 serotypes were the most frequently found IPD serotypes in children aged $<18$ years, especially serotype $19 \mathrm{~A}$ in children aged $<2$ years, serotype 3 in the 2-4 years age group and serotype 1 in the 5-17 years age group.

Non-PCV13 serotypes were the main cause of meningitis, occult bacteraemia and other clinical presentations, while PCV13 serotypes were mainly responsible for pneumonia.

PCV13 and non-PCV13 cases presented high frequency of non-susceptibility to penicillin. Non-susceptibility to both penicillin and cefotaxime was associated with serotypes $19 \mathrm{~A}$ and 14 , and serotype 19A was associated with resistance to penicillin.

The non-susceptibility to antibiotics of non-PCV13 serotypes is a worrisome fact and should be monitored to apply the appropriate disease prevention strategies.

\section{List Of Abbreviations}

IPD: Invasive pneumococcal disease; PCV7: 7-valent pneumococcal conjugate vaccine; PCV13: 13-valent pneumococcal conjugate vaccine; NonPCV13: serotypes not included in 13-valent pneumococcal conjugate vaccine; RT-PCR: real-time polymerase chain reaction; ICU: intensive care unit; Cl: confidence intervals. 


\section{Declarations}

Ethics approval and consent to participate

Informed consent was obtained from all individual participants included in the study. Written informed consent was obtained from a parent o guardian for participants under 16 years old. The study complies with the principles of the Declaration of Helsinki and the legal structure with respect to international human rights and biomedicine and protection of personal data laws. The Ethics Committee of Hospital Sant Joan de Déu approved the study.

Consent for publication

Not applicable

Availability of data and material

The dataset supporting the conclusions of this article is included within the article.

Competing interests

JJ Garcia-Garcia has received honoraria for speaking at symposia (Pfizer and GSK), and Financial support for attending symposia (Pfizer). All outside the submitted work.

C Muñoz-Almagro reports travel grants from Pfizer, research grants from BioMerieux, Stat DX and Instituto de Salud Carlos III, personal fees from GSK as consultor advisor board and honoraria for speaking at symposium from Roche and Biomerieux. All outside the submitted work.

For de remaining author none were declared.

Funding

This work was supported by the Plan Nacional I+D+I, Instituto de Salud Carlos III -Subdirección General de Evaluación y Fomento de la Investigación Sanitaria (Projects: PI11/02081 and PI11/02345) and cofounded by the Fondo Europeo de Desarrollo Regional (FEDER) (their funding role was for serotyping study and for participating in congresses) and the Agència de gestió d'ajuts universitaris i recerca (AGAUR: grant number 2017 SGR 1342 ) (its funding role was for English manuscript revision).

\section{Authors' contributions}

$\mathrm{Cl}$ made substantial contributions to the data collection process and to the conception, design, analysis, and interpretation of data. $\mathrm{Cl}$ and PC drafted and revised the manuscript and gave the final approval of the version to be published, and $\mathrm{Cl}$ is the corresponding author. SH, JJGG, CE, FMLL, ADC, JMO, ASR, MFS, SGP, GC, AMP, SU, MC, CMA, LS, and AD made contributions to the interpretation of the data, provided comments on the draft and have read and approved the final version.

Acknowledgements

Not Applicable

\section{References}

1. Wahl B, O’Brien KL, Greenbaum A, Liu L, Chu Y, Black R, et al. Global burden of Streptococcus pneumoniae in children younger than 5 years in the era of pneumococcal conjugate vaccines (PCV): 2000-2015. In: 10th International Symposium on Pneumococci and Pneumococcal Diseases (ISPPD-10), July 26 to 30, 2016. Glasgow, Scotland; 2016.

2. Whitney CG, Farley MM, Hadler J, Harrison LH, Bennett NM, Lynfield R, et al. Decline in invasive pneumococcal disease after the introduction of proteinpolysaccharide conjugate vaccine. N Engl J Med. 2003; 348:1737-46.

3. Rückinger S, van der Linden M, Reinert RR, von Kries R, Burckhardt F, Siedler A. Reduction in the incidence of invasive pneumococcal disease after general vaccination with 7-valent pneumococcal conjugate vaccine in Germany. Vaccine. 2009; 27:4136-41.

4. Harboe ZB, Valentiner-Branth P, Benfield TL, Christensen JJ, Andersen PH, Howitz M, et al. Early effectiveness of heptavalent conjugate pneumococcal vaccination on invasive pneumococcal disease after the introduction in the Danish childhood immunization programme. Vaccine. 2010; 28:2642-47.

5. Roche PW, Krause V, Cook H, Barralet J, Coleman D, Sweeny A et al. Invasive pneumococcal disease in Australia, 2006. Commun Dis Intell. 2008; 32 : $18-30$.

6. Centers for Disease Control and Prevention. Licensure of a 13-Valent pneumococcal conjugate vaccine (PCV13) and recommendations for use among children-advisory committee on immunization practices (ACIP) MMWR. 2010; 59 (9):258-61.

7. Tin Tin Htar M, Christopoulou D, Schmitt HJ. Pneumococcal serotype evolution in Western Europe. BMC Infect Dis. 2015;15:419.

8. Bernaola E, Giménez F, Baca M, De Juan F, Díez J, Garces M et al. Vaccination schedule of the Spanish association of pediatrics: recommendations 2009. An Pediatr (Barc).2009;70:72-82.

9. Ciruela P, Soldevila N, Hernández S, Selva L, de Sevilla MF, Garcia-Garcia JJ et al. Risk factors for invasive pneumococcal disease in a community with a high proportion of non-vaccine serotypes. Vaccine. 2013; 31: 960-6.

Page 6/13 
10. Salleras L, Domínguez A, Ciruela P, Izquierdo C, Navas E, Torner $\mathrm{N}$ et al. Changes in serotypes causing invasive pneumococcal disease (2005-2007 vs 1997-1999) in children under 2 years of age in a population with intermediate coverage of the 7-valent pneumococcal conjugated vaccine. Clin Microbiol Infect. 2009; 15:997-1001.

11. Generalitat de Catalunya, Departament de Salut (2016) New vaccine schedule.http://canalsalut.gencat.cat/ca/salut-a-z/v/vacunacions/calendari-devacunacions/. Accesed July 2019.

12. Savulescu C, Krizova P, Lepoutre A, Mereckiene J, Vestrheim DF, Ciruela P, et al. Effect of high valency pneumococcal conjugate vaccines on invasive pneumococcal disease in children in SpIDnet countries: an observational multicentre study. Lancet Respir Med. 2017; 5:648-56.

13. Tomczyk S, Lynfield R, Schaffner W, Reingold A, Miller L, Petit S, et al. Prevention of antibiotic-nonsusceptible invasive pneumococcal disease with the 13-valent pneumococcal conjugate vaccine. Clin Infect Dis. 2016; 62:1119-25.

14. Janoir C, Lepoutre A, Gutmann L, Varon E. Insight into resistance phenotypes of emergent non 13-valent pneumococcal conjugate vaccine type pneumococci isolated from invasive disease after 13-valent pneumococcal conjugate vaccine implementation in France. Open Forum Infect Dis. 2016; 3:ofw020.

15. Selva L, del Amo E, Brotons P, Muñoz-Almagro C. Rapid and easy identification of capsular serotypes of Streptococcus pneumoniae by use of fragment analysis by automated fluorescence-based capillary electrophoresis. J Clin Microbiol. 2012; 50:3451-7.

16. Fenoll A, Jado I, Vicioso D, Casal J. Dot blot assay for the serotyping of pneumococci. J Clin Microbiol. 1997; 35:764-6.

17. The European Committee on Antimicrobial Susceptibility Testing. Breakpoint tables for interpretation of MICs and zone diameters. Version 8.1, 2018. Accessed July 2019 http://www.eucast.org/clinical_breakpoints/.

18. Sheskin DJ. Handbook of parametric and nonparametric statistical procedures. 3rd ed. Boca Raton: Chapman \& Hall /CRC; 2004.

19. Ciruela P, Izquierdo C, Broner S, Muñoz-Almagro C, Hernandez S, Ardanuy C et al. The changing epidemiology of invasive pneumococcal disease after PCV13 vaccination in a country with intermediate vaccination coverage. Vaccine 2018; 36:7744-52.

20. Makwana A, Sheppard C, Borrow R, Fry N, Andrews NJ, Ladhani SN. Characteristics of children with invasive pneumococcal disease after the Introduction of the 13-valent pneumococcal conjugate vaccine in England and Wales, 2010-2016. Pediatr Infect Dis J. 2018;37:697-703.

21. Picazo J, Ruiz-Contreras J, Casado-Flores J, Negreira S, Baquero F, Hernandez-Sampelayo T, et al. Effect of the different 13-valent pneumococcal conjugate vaccination uptakes on the invasive pneumococcal disease in children: analysis of a hospital-based and population-based surveillance study in Madrid, Spain, 2007-2015. PLoS One. 2017; 12:e0172222.

22. Ceyhan M, Ozsurekci Y, Gürler N, Öksüz L, Aydemir S, Ozkan S, et al. Serotype distribution of Streptococcus pneumoniae in children with invasive diseases in Turkey: 2008-2014. Human Vaccines \& Immunotherapeutics. 2016; 12: 308-13.

23. Camilli R, D’Ambrosio F, Del Grosso M, Pimentel de Araujo F, Caporali MG, Del Manso M, et al. Impact of pneumococcal conjugate vaccine (PCV7 and PCV13) on pneumococcal invasive diseases in Italian children and insight into evolution of pneumococcal population structure. Vaccine. 2017; 35:4587-93.

24. Selva L, Ciruela P, Esteva C, de Sevilla MF, Codina G, Hernandez S et al. Serotype 3 is a common serotype causing invasive pneumococcal disease in children less than 5 years old, as identified by real-time PCR. Eur J Clin Microbiol Infect Dis. 2012; 31:1487-95.

25. Skoczyńska A, Kuch A, Sadowy E. Waśko I, Markowska M, Ronkiewicz P, et al. Recent trends in epidemiology of invasive pneumococcal disease in Poland. Eur J Clin Microbiol Infect Dis. 2015; 34:779-87

26. Picazo J, Ruiz-Contreras J, Casado-Flores J, Negreira S, Garcia de Miguel M, Hernandez-Sampelayo T et al. Expansion of serotype coverage in the universal pediatric vaccination calendar: short-term effects on age- and serotype-dependent incidence of invasive pneumococcal clinical presentations in Madrid, Spain. Clinical and Vaccine Immunology. 2013; 20:1524-30.

27. Van der Linden M, Perniciaro S, Imöhl M. Increase of serotypes 15A and 23B in IPD in Germany in the PCV13 vaccination era. BMC Infect Dis. 2015;15: 207 10.1186/s12879-015-0941-9.

\section{Tables}

Table 1. Demographic characteristics and clinical presentation in children $\leq 17$ years with IPD. 


\begin{tabular}{|c|c|}
\hline & $18(10)$ \\
\hline & (253 cases) \\
\hline \multicolumn{2}{|l|}{ Age group } \\
\hline$<2$ years & $87(34.4)$ \\
\hline 2-4 years & $98(38.7)$ \\
\hline 5-17 years & $68(26.9)$ \\
\hline \multicolumn{2}{|l|}{ Sex } \\
\hline Male & $151(59.7)$ \\
\hline \multicolumn{2}{|l|}{ Seasonality } \\
\hline October-March & $182(72.0)$ \\
\hline \multicolumn{2}{|l|}{ Diagnosis } \\
\hline Culture only & $62(24.5)$ \\
\hline PCR only & $122(48.2)$ \\
\hline Culture + PCR & $69(27.3)$ \\
\hline \multicolumn{2}{|l|}{ Clinical presentation } \\
\hline Overall pneumonia & $187(73.9)$ \\
\hline Complicated pneumonia & $150(80.2)$ \\
\hline Occult bacteraemia & $25(9.9)$ \\
\hline Meningitis & $18(7.1)$ \\
\hline Other forms $^{\mathrm{a}}$ & $23(9.1)$ \\
\hline \multicolumn{2}{|l|}{ ICU admission } \\
\hline Yes & $49(19.4)$ \\
\hline \multicolumn{2}{|l|}{ Underlying medical condition } \\
\hline Yes & $15(6.0)$ \\
\hline \multirow{2}{*}{\multicolumn{2}{|c|}{$\begin{array}{l}\text { Case fatality rate } \\
\text { Yes }\end{array}$}} \\
\hline & \\
\hline \multicolumn{2}{|l|}{ Serotype groups } \\
\hline PCV13 & $163(64.4)$ \\
\hline Non-PCV13 & $90(35.6)$ \\
\hline \multicolumn{2}{|l|}{ Pneumococcal vaccination* } \\
\hline PCV7 ( $\geq 1$ dose) & $33(13.1)$ \\
\hline PCV10 ( $\geq 1$ dose) & $7(2.8)$ \\
\hline PCV13 ( $\geq 1$ dose) & $83(32.9)$ \\
\hline Non-vaccinated & $129(51.2)$ \\
\hline \multicolumn{2}{|c|}{ Antimicrobial susceptibility ( $n=122$ strains) } \\
\hline Penicillin non-susceptible** & $44(36.1)$ \\
\hline Cefotaxime non-susceptible & $20(16.4)$ \\
\hline
\end{tabular}

*1 missing case $* * 4$ cases were penicillin resistant.

${ }^{\text {a }}$ Other forms: septic shock ( 7 cases), mastoiditis ( 7 cases), osteoarticular infection (6 cases), orbital cellulitis (2 cases), pancreatitis (1 case).

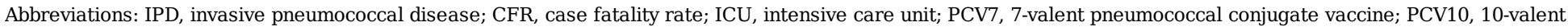
pneumococcal conjugate vaccine; PCV13, 13-valent pneumococcal conjugate vaccine. 
Table 2. Distribution of Streptococcus pneumoniae serotypes causing IPD in children aged $\leq 17$ years by clinical presentation.

\begin{tabular}{|c|c|c|c|c|c|c|c|c|c|c|c|c|c|c|c|c|c|}
\hline \multirow[t]{2}{*}{ Serotype } & \multicolumn{2}{|c|}{ Total } & \multicolumn{3}{|c|}{ Meningitis } & \multicolumn{3}{|c|}{ Overall pneumonia } & \multicolumn{3}{|c|}{ Complicated pneumonia } & \multicolumn{3}{|c|}{ Occult bacteraemia } & \multicolumn{3}{|c|}{ Others } \\
\hline & $\mathrm{n}$ & $\%$ & $\mathrm{n}$ & $\%$ & $\begin{array}{c}\mathrm{p} \\
\text { value* }\end{array}$ & $\mathrm{n}$ & $\%$ & p value* & $\mathrm{n}$ & $\%$ & $\begin{array}{c}\mathrm{p} \\
\text { value* }\end{array}$ & $\mathrm{n}$ & $\%$ & $\begin{array}{c}\mathrm{p} \\
\text { value* }\end{array}$ & $\mathrm{n}$ & $\%$ & $\begin{array}{c}\mathrm{p} \\
\text { value* }\end{array}$ \\
\hline \multicolumn{18}{|l|}{ PCV13 } \\
\hline 1 & 48 & 19.0 & 0 & 0.0 & - & 47 & 97.9 & $<0.001$ & 41 & 85.4 & $<0.001$ & 0 & 0.0 & - & 1 & 2.1 & ns \\
\hline 3 & 53 & 20.9 & 1 & 1.9 & ns & 49 & 92.5 & 0.001 & 44 & 83.0 & $<0.001$ & 0 & 0.0 & - & 3 & 5.7 & ns \\
\hline 4 & 2 & 0.8 & 0 & 0.0 & - & 1 & 50.0 & ns & 0 & 0.0 & - & 1 & 50.0 & ns & 0 & 0.0 & - \\
\hline $6 A^{a}$ & 3 & 1.2 & 0 & 0.0 & - & 3 & 100.0 & ns & 3 & 100.0 & ns & 0 & 0.0 & - & 0 & 0.0 & - \\
\hline $6 B$ & 2 & 0.8 & 0 & 0.0 & - & 2 & 100.0 & ns & 1 & 50.0 & ns & 0 & 0.0 & - & 0 & 0.0 & - \\
\hline $7 \mathrm{~F}^{\mathrm{a}}$ & 9 & 3.6 & 0 & 0.0 & - & 9 & 100.0 & ns & 9 & 100.0 & ns & 0 & 0.0 & - & 0 & 0.0 & - \\
\hline $9 \mathrm{~V}^{\mathrm{a}}$ & 6 & 2.4 & 0 & 0.0 & - & 4 & 66.7 & ns & 4 & 66.7 & ns & 2 & 33.3 & ns & 0 & 0.0 & - \\
\hline 14 & 15 & 5.9 & 1 & 6.7 & ns & 12 & 80.0 & ns & 9 & 60.0 & ns & 1 & 6.7 & ns & 1 & 6.7 & ns \\
\hline $18 \mathrm{C}$ & 2 & 0.8 & 0 & 0.0 & - & 0 & 0.0 & - & 0 & 0.0 & - & 2 & 100.0 & 0.009 & 0 & 0.0 & - \\
\hline $19 A$ & 17 & 6.7 & 1 & 5.9 & ns & 15 & 88.2 & ns & 12 & 70.6 & ns & 0 & 0.0 & - & 1 & 5.9 & ns \\
\hline $19 \mathrm{~F}^{\mathrm{a}}$ & 5 & 2.0 & 1 & 20.0 & ns & 1 & 20.0 & ns & 0 & 0.0 & - & 2 & 40.0 & ns & 1 & 20.0 & ns \\
\hline $23 F$ & 1 & 0.4 & 0 & 0.0 & - & 0 & 0.0 & - & 0 & 0.0 & - & 1 & 100.0 & ns & 0 & 0.0 & - \\
\hline \multicolumn{18}{|c|}{ Non-PCV13 } \\
\hline $6 C$ & 1 & 0.4 & 0 & 0.0 & - & 1 & 100.0 & ns & 0 & 0.0 & - & 0 & 0.0 & - & 0 & 0.0 & - \\
\hline 8 & 2 & 0.8 & 0 & 0.0 & - & 1 & 50.0 & & 1 & 50.0 & ns & 0 & 0.0 & - & 1 & 50.0 & ns \\
\hline $10 \mathrm{~A}$ & 6 & 2.4 & 1 & 16.7 & ns & 1 & 16.7 & ns & 0 & 0.0 & - & 3 & 50.0 & 0.014 & 1 & 16.7 & ns \\
\hline $11 \mathrm{~A}$ & 5 & 2.0 & 0 & 0.0 & - & 3 & 60.0 & ns & 1 & 20.0 & ns & 0 & 0.0 & - & 2 & 40.0 & ns \\
\hline $12 \mathrm{~F}$ & 3 & 1.2 & 0 & 0.0 & - & 1 & 33.3 & ns & 1 & 33.3 & ns & 2 & 66.7 & 0.026 & 0 & 0.0 & - \\
\hline 13 & 1 & 0.4 & 1 & 100.0 & ns & 0 & 0.0 & - & 0 & 0.0 & - & 0 & 0.0 & - & 0 & 0.0 & - \\
\hline $15 A$ & 3 & 1.2 & 1 & 33.3 & ns & 0 & 0.0 & ns & 0 & 0.0 & - & 1 & 33.3 & ns & 1 & 33.3 & ns \\
\hline 15B & 2 & 0.8 & 1 & 50.0 & ns & 0 & 0.0 & - & 0 & 0.0 & - & 1 & 50.0 & ns & 0 & 0.0 & - \\
\hline $15 \mathrm{C}$ & 1 & 0.4 & 0 & 0.0 & - & 0 & 0.0 & - & 0 & 0.0 & - & 1 & 100.0 & ns & 0 & 0.0 & - \\
\hline $16 \mathrm{~F}$ & 2 & 0.8 & 1 & 50.0 & ns & 0 & 0.0 & - & 0 & 0.0 & - & 1 & 50.0 & ns & 0 & 0.0 & - \\
\hline $22 F$ & 2 & 0.8 & 1 & 50.0 & ns & 0 & 0.0 & - & 0 & 0.0 & - & 0 & 0.0 & - & 1 & 50.0 & ns \\
\hline $23 \mathrm{~A}$ & 1 & 0.4 & 0 & 0.0 & - & 0 & 0.0 & - & 0 & 0.0 & - & 0 & 0.0 & - & 1 & 100.0 & ns \\
\hline 23B & 3 & 1.2 & 0 & 0.0 & - & 1 & 33.3 & ns & 1 & 33.3 & ns & 1 & 33.3 & ns & 1 & 33.3 & ns \\
\hline 24 & 2 & 0.8 & 0 & 0.0 & - & 1 & 50.0 & ns & 0 & 0.0 & - & 1 & 50.0 & ns & 0 & 0.0 & - \\
\hline $24 \mathrm{~A}$ & 2 & 0.8 & 1 & 50.0 & ns & 1 & 50.0 & ns & 0 & 0.0 & - & 0 & 0.0 & - & 0 & 0.0 & - \\
\hline $24 \mathrm{~F}$ & 6 & 2.4 & 1 & 16.7 & ns & 4 & 66.7 & ns & 2 & 33.3 & ns & 1 & 16.7 & ns & 0 & 0.0 & \\
\hline $25 \mathrm{~F}$ & 1 & 0.4 & 0 & 0.0 & - & 0 & 0.0 & - & 0 & 0.0 & - & 1 & 100.0 & ns & 0 & 0.0 & - \\
\hline 27 & 2 & 0.8 & 0 & 0.0 & - & 0 & 0.0 & - & 0 & 0.0 & - & 1 & 50.0 & ns & 1 & 50.0 & ns \\
\hline 31 & 2 & 0.8 & 0 & 0.0 & - & 0 & 0.0 & - & 0 & 0.0 & - & 1 & 50.0 & ns & 1 & 50.0 & ns \\
\hline $33 F$ & 2 & 0.8 & 0 & 0.0 & - & 1 & 50.0 & ns & 0 & 0.0 & - & 0 & 0.0 & - & 1 & 50.0 & ns \\
\hline 35B & 1 & 0.4 & 0 & 0.0 & - & 0 & 0.0 & - & 0 & 0.0 & - & 1 & 100.0 & ns & 0 & 0.0 & - \\
\hline $35 \mathrm{~F}$ & 1 & 0.4 & 1 & 100.0 & ns & 0 & 0.0 & - & 0 & 0.0 & - & 0 & 0.0 & - & 0 & 0.0 & - \\
\hline 38 & 2 & 0.8 & 0 & 0.0 & - & 1 & 50.0 & ns & 1 & 50.0 & ns & 0 & 0.0 & - & 1 & 50.0 & ns \\
\hline ONV & 37 & 14.6 & 5 & 13.5 & - & 28 & 75.7 & - & 20 & 54.1 & - & 0 & 0.0 & - & 4 & 10.8 & - \\
\hline Total & 253 & 100.0 & 18 & 7.1 & - & 187 & 73.9 & - & 150 & 59.3 & - & 25 & 9.9 & - & 23 & 9.1 & - \\
\hline
\end{tabular}

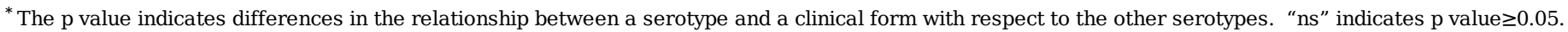

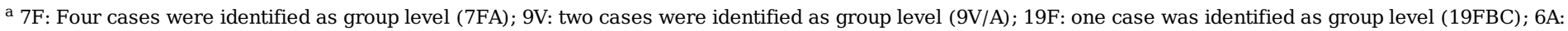
two cases were identified as group level (6A/6C).

Abbreviations: IPD, invasive pneumococcal disease; ONV, Other non-vaccine serotypes. 
Table 3. Distribution of Streptococcus pneumoniae serotype groups causing IPD in children $\leq 17$ years by clinical presentation and age group.

\begin{tabular}{|c|c|c|c|c|c|c|c|c|c|c|c|c|c|c|c|c|c|c|}
\hline \multirow{3}{*}{$\begin{array}{l}\begin{array}{l}\text { Age } \\
\text { groups }\end{array} \\
0-17 \\
\text { years }\end{array}$} & \multirow{3}{*}{$\begin{array}{l}\text { Serotype } \\
\text { group }\end{array}$} & \multirow{2}{*}{\multicolumn{2}{|c|}{ Total }} & \multicolumn{12}{|c|}{$\begin{array}{c}\text { Clinical presentation } \\
\end{array}$} & \multirow{2}{*}{\multicolumn{3}{|c|}{ Other forms }} \\
\hline & & & & \multicolumn{3}{|c|}{ Meningitis } & \multicolumn{3}{|c|}{ Overall pneumonia } & \multicolumn{3}{|c|}{$\begin{array}{c}\text { Complicated } \\
\text { pneumonia }\end{array}$} & \multicolumn{2}{|c|}{ Occult bacteraemia } & \multirow[b]{2}{*}{$\begin{array}{c}\mathrm{p} \\
\text { value* }\end{array}$} & & & \\
\hline & & $\mathrm{n}$ & $\%$ & $\mathrm{n}$ & $\%$ & $\begin{array}{c}\mathrm{p} \\
\text { value* }\end{array}$ & $\mathrm{n}$ & $\%$ & $\begin{array}{c}\mathrm{p} \\
\text { value* }\end{array}$ & $\mathrm{n}$ & $\%$ & $\begin{array}{c}\mathrm{p} \\
\text { value* }\end{array}$ & $\mathrm{n}$ & $\%$ & & $\mathrm{n}$ & $\%$ & p value* \\
\hline & PCV13 & 163 & 64.4 & 4 & 2.5 & & 143 & 87.7 & $<0.001$ & 123 & 75.5 & $<0.001$ & 9 & 5.5 & & 7 & 4.3 & \\
\hline & $\begin{array}{l}\text { Non- } \\
\text { PCV13 }\end{array}$ & 90 & 35.6 & 14 & 15.6 & $<0.001$ & 44 & 48.9 & & 27 & 30.0 & & 16 & 17.8 & 0.002 & 16 & 17.8 & $<0.001$ \\
\hline & Total & 253 & 100.0 & 18 & 7.1 & - & 187 & 73.9 & - & 150 & 59.3 & - & 25 & 9.9 & - & 23 & 9.1 & - \\
\hline \multicolumn{19}{|l|}{$\begin{array}{l}<2 \\
\text { years }\end{array}$} \\
\hline & PCV13 & 39 & 44.8 & 3 & 7.7 & - & 29 & 74.4 & $<0.001$ & 23 & 59.0 & 0.001 & 4 & 10.3 & - & 3 & 7.7 & - \\
\hline & $\begin{array}{l}\text { Non- } \\
\text { PCV13 }\end{array}$ & 48 & 55.2 & 10 & 20.8 & ns & 17 & 35.4 & - & 11 & 22.9 & - & 12 & 25 & ns & 9 & 18.8 & ns \\
\hline & Total & 87 & 100.0 & 13 & 14.9 & & 46 & 52.9 & & 34 & 39.1 & & 16 & 18.4 & & 12 & 13.8 & \\
\hline \multicolumn{19}{|l|}{$\begin{array}{l}2-4 \\
\text { years }\end{array}$} \\
\hline & PCV13 & 71 & 72.4 & 0 & 0 & - & 65 & 91.5 & 0.004 & 57 & 80.3 & $<0.001$ & 4 & 5.6 & - & 2 & 2.8 & - \\
\hline & $\begin{array}{l}\text { Non- } \\
\text { PCV13 }\end{array}$ & 27 & 27.6 & 4 & 14.8 & 0.005 & 18 & 66.7 & - & 10 & 37.0 & - & 2 & 7.4 & ns & 3 & 11.1 & ns \\
\hline & Total & 98 & 100.0 & 4 & 4.1 & & 83 & 84.7 & & 67 & 68.4 & & 6 & 6.1 & & 5 & 5.1 & \\
\hline \multicolumn{19}{|l|}{$\begin{array}{c}5-17 \\
\text { years }\end{array}$} \\
\hline & PCV13 & 53 & 77.9 & 1 & 1.9 & ns & 49 & 92.5 & 0.006 & 43 & 81.1 & 0.002 & 1 & 1.9 & - & 2 & 3.8 & - \\
\hline & $\begin{array}{l}\text { Non- } \\
\text { PCV13 }\end{array}$ & 15 & 22.1 & 0 & 0 & - & 9 & 60.0 & - & 6 & 40.0 & - & 2 & 13.3 & ns & 4 & 26.7 & 0.018 \\
\hline & Total & 68 & 100.0 & 1 & 1.5 & & 58 & 85.3 & & 49 & 72.1 & & 3 & 4.4 & & 6 & 8.8 & \\
\hline
\end{tabular}

* The p value indicates differences in the relationship between a serotype group and a clinical form with respect to the other serotypes.

"ns" indicates p value $\geq 0.05$. 
Table 4: Distribution of Streptococcus pneumoniae serotypes causing IPD in children $\leq 17$ years by non-susceptibility to antibiotics.

\begin{tabular}{|c|c|c|c|c|c|c|c|c|c|c|c|c|c|c|c|}
\hline \multirow[t]{2}{*}{ Serotype } & \multirow{2}{*}{$\begin{array}{c}\text { Total cases } \\
\mathrm{n}\end{array}$} & \multicolumn{2}{|c|}{ Analyzed cases } & \multicolumn{3}{|c|}{ Penicillin $>0.06^{\mathrm{a}}$} & \multicolumn{3}{|c|}{ Penicillin $>2^{\mathrm{b}}$} & \multicolumn{3}{|c|}{ Cefotaxime $>0.5^{\mathrm{C}}$} & \multicolumn{3}{|c|}{ Penicillin $>0.06+$ Cefotaxime $>0.5^{\mathrm{d}}$} \\
\hline & & $\mathrm{n}$ & $\%$ & $\mathrm{n}$ & $\%$ & p value* & $\mathrm{n}$ & $\%$ & $\begin{array}{c}\mathrm{p} \\
\text { value* }^{*}\end{array}$ & $\mathrm{n}$ & $\%$ & $\begin{array}{c}\mathrm{p} \\
\text { value* }\end{array}$ & $\mathrm{n}$ & $\%$ & $\begin{array}{c}\mathrm{p} \\
\text { value } *\end{array}$ \\
\hline \multicolumn{16}{|l|}{ PCV13 } \\
\hline 1 & 48 & 27 & 56.3 & 0 & 0.0 & - & 0 & 0.0 & - & 0 & 0.0 & - & 0 & 0.0 & - \\
\hline 3 & 53 & 6 & 11.3 & 0 & 0.0 & - & 0 & 0.0 & - & 0 & 0.0 & - & 0 & 0.0 & - \\
\hline 4 & 2 & 2 & 100.0 & 0 & 0.0 & - & 0 & 0.0 & - & 0 & 0.0 & - & 0 & 0.0 & - \\
\hline $6 B$ & 2 & 1 & 50.0 & 1 & 100 & ns & 0 & 0.0 & - & 0 & 0.0 & - & 0 & 0.0 & - \\
\hline $7 \mathrm{~F}^{\mathrm{e}}$ & 9 & 1 & 11.1 & 0 & 0.0 & - & 0 & 0.0 & - & 0 & 0.0 & - & 0 & 0.0 & - \\
\hline $9 \mathrm{~V}^{\mathrm{e}}$ & 6 & 5 & 83.3 & 1 & 20.0 & ns & 0 & 0.0 & - & 1 & 20.0 & ns & 1 & 20.0 & ns \\
\hline 14 & 15 & 11 & 73.3 & 11 & 100.0 & $<0.001$ & 0 & 0.0 & - & 9 & 81.8 & $<0.001$ & 9 & 81.8 & $<0.001$ \\
\hline $18 \mathrm{C}$ & 2 & 2 & 100.0 & 0 & 0.0 & - & 0 & 0.0 & - & 0 & 0.0 & - & 0 & 0.0 & - \\
\hline $19 \mathrm{~A}$ & 17 & 11 & 64.7 & 10 & 90.9 & $<0.001$ & 3 & 27.3 & 0.002 & 6 & 54.5 & 0.003 & 6 & 54.5 & 0.003 \\
\hline $19 \mathrm{~F}^{\mathrm{e}}$ & 5 & 4 & 80.0 & 3 & 75.0 & ns & 1 & 25.0 & ns & 1 & 25.0 & ns & 1 & 25.0 & ns \\
\hline \multicolumn{16}{|l|}{ Non-PCV13 } \\
\hline $6 C$ & 1 & 1 & 100.0 & 0 & 0.0 & - & 0 & 0.0 & - & 0 & 0.0 & - & 0 & 0.0 & - \\
\hline 8 & 2 & 2 & 100.0 & 0 & 0.0 & - & 0 & 0.0 & - & 0 & 0.0 & - & 0 & 0.0 & - \\
\hline $10 \mathrm{~A}$ & 6 & 5 & 83.3 & 0 & 0.0 & - & 0 & 0.0 & - & 0 & 0.0 & & 0 & 0.0 & \\
\hline $24 \mathrm{~F}$ & 6 & 6 & 100.0 & 5 & 83.3 & 0.023 & 0 & 0.0 & - & 0 & 0.0 & - & 0 & 0.0 & - \\
\hline $11 \mathrm{~A}$ & 5 & 5 & 100.0 & 3 & 60.0 & ns & 0 & 0.0 & - & 3 & 60.0 & 0.031 & 3 & 60.0 & 0.031 \\
\hline $12 F$ & 3 & 3 & 100.0 & 0 & 0.0 & - & 0 & 0.0 & - & 0 & 0.0 & - & 0 & 0.0 & - \\
\hline 13 & 1 & 1 & 100.0 & 0 & 0.0 & - & 0 & 0.0 & - & 0 & 0.0 & - & 0 & 0.0 & - \\
\hline $15 \mathrm{~A}$ & 3 & 3 & 100.0 & 1 & 33.3 & ns & 0 & 0.0 & - & 0 & 0.0 & - & 0 & 0.0 & - \\
\hline $15 B$ & 2 & 2 & 100.0 & 0 & 0.0 & - & 0 & 0.0 & - & 0 & 0.0 & - & 0 & 0.0 & - \\
\hline $15 C$ & 1 & 1 & 100.0 & 0 & 0.0 & - & 0 & 0.0 & - & 0 & 0.0 & - & 0 & 0.0 & - \\
\hline $16 \mathrm{~F}$ & 2 & 2 & 100.0 & 1 & 50.0 & ns & 0 & 0.0 & - & 0 & 0.0 & - & 0 & 0.0 & - \\
\hline $22 \mathrm{~F}$ & 2 & 2 & 100.0 & 0 & 0.0 & - & 0 & 0.0 & - & 0 & 0.0 & - & 0 & 0.0 & - \\
\hline 23B & 3 & 3 & 100.0 & 3 & 100.0 & 0.045 & 0 & 0.0 & - & 0 & 0.0 & - & 0 & 0.0 & - \\
\hline 27 & 2 & 2 & 100.0 & 1 & 50.0 & ns & 0 & 0.0 & - & 0 & 0.0 & - & 0 & 0.0 & - \\
\hline 31 & 2 & 2 & 100.0 & 0 & 0.0 & - & 0 & 0.0 & - & 0 & 0.0 & - & 0 & 0.0 & - \\
\hline $33 F$ & 2 & 2 & 100.0 & 0 & 0.0 & - & 0 & 0.0 & - & 0 & 0.0 & - & 0 & 0.0 & - \\
\hline $35 B$ & 1 & 1 & 100.0 & 0 & 0.0 & - & 0 & 0.0 & - & 0 & 0.0 & - & 0 & 0.0 & - \\
\hline $35 \mathrm{~F}$ & 1 & 1 & 100.0 & 0 & 0.0 & - & 0 & 0.0 & - & 0 & 0.0 & - & 0 & 0.0 & - \\
\hline 38 & 2 & 2 & 100.0 & 0 & 0.0 & - & 0 & 0.0 & - & 0 & 0.0 & - & 0 & 0.0 & - \\
\hline PCV13 & 163 & 72 & 44.2 & 26 & 36.1 & ns & 4 & 5.6 & ns & 17 & 23.6 & 0.010 & 17 & 23.6 & 0.010 \\
\hline Non-PCV13 & 90 & 50 & 55.6 & 18 & 36.0 & & 0 & 0.0 & & 3 & 6.0 & & 3 & 6.0 & \\
\hline Total & 253 & 122 & 48.2 & 44 & 36.1 & -- & 4 & 3.3 & -- & 20 & 16.4 & -- & 20 & 16.4 & -- \\
\hline
\end{tabular}

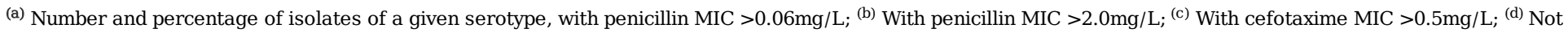
susceptible to both penicillin and cefotaxime.

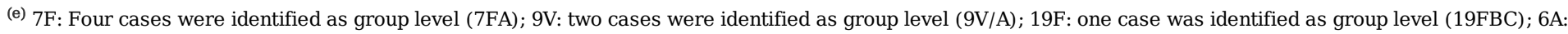
two cases were identified as group level (6A/6C).

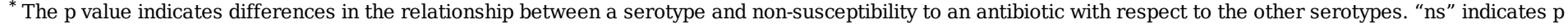
value $\geq 0.05$.

Abbreviations: IPD, invasive pneumococcal disease; ONV, Other non-vaccine serotypes.

Figures 


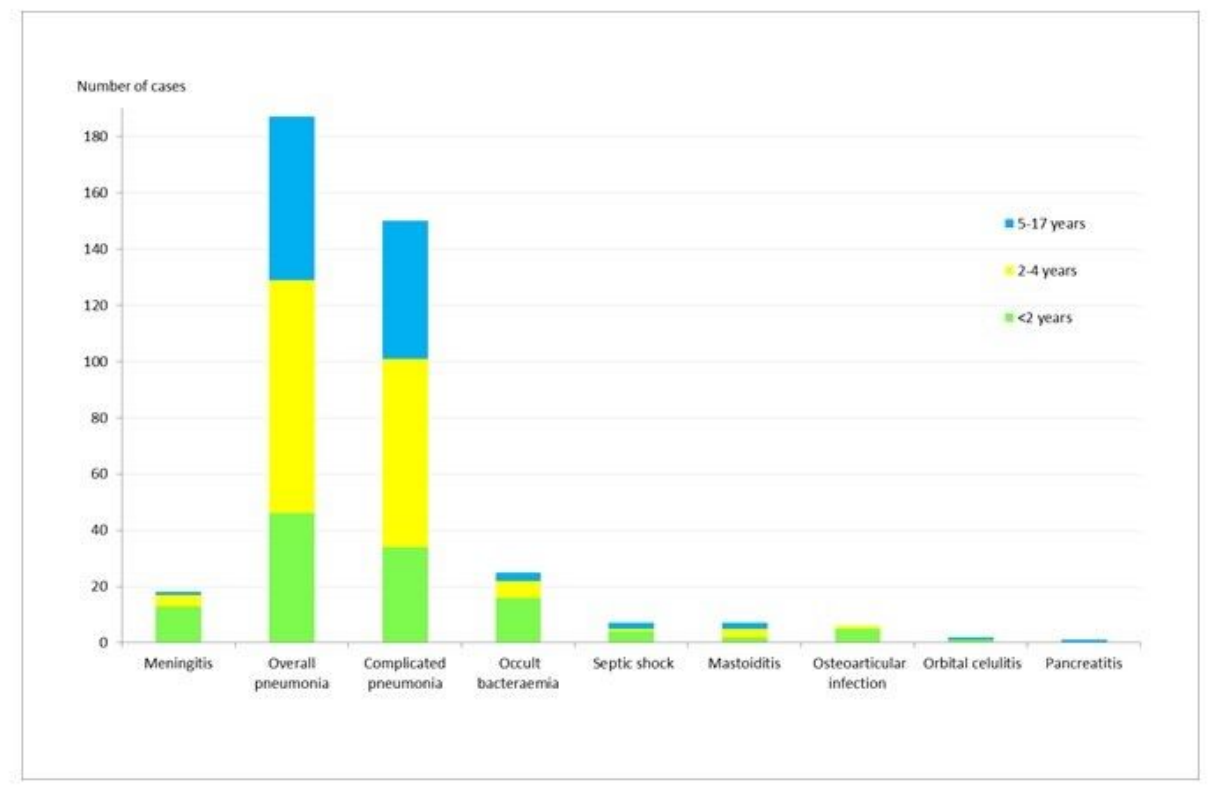

\section{Figure 1}

Distribution of IPD clinical presentation in children $\leq 17$ years by age group. IPD: invasive pneumococcal disease

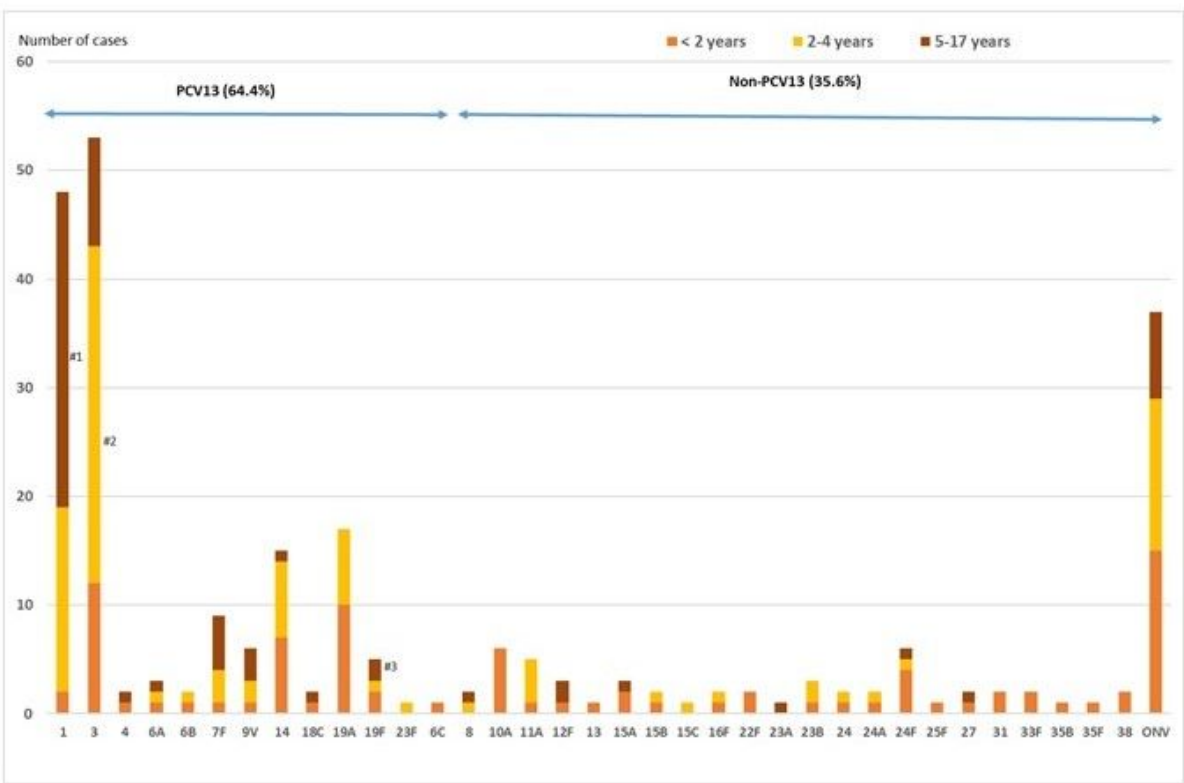

\section{Figure 2}

Distribution of Streptococcus pneumoniae serotypes causing IPD in children $\leq 17$ years by age groups. IPD: invasive pneumococcal disease. ONV: Other non-vaccine serotypes. \#1: $p<0.001 ; \# 2$ : $p=0.001 ; \# 3$ : $p=0.028$ 

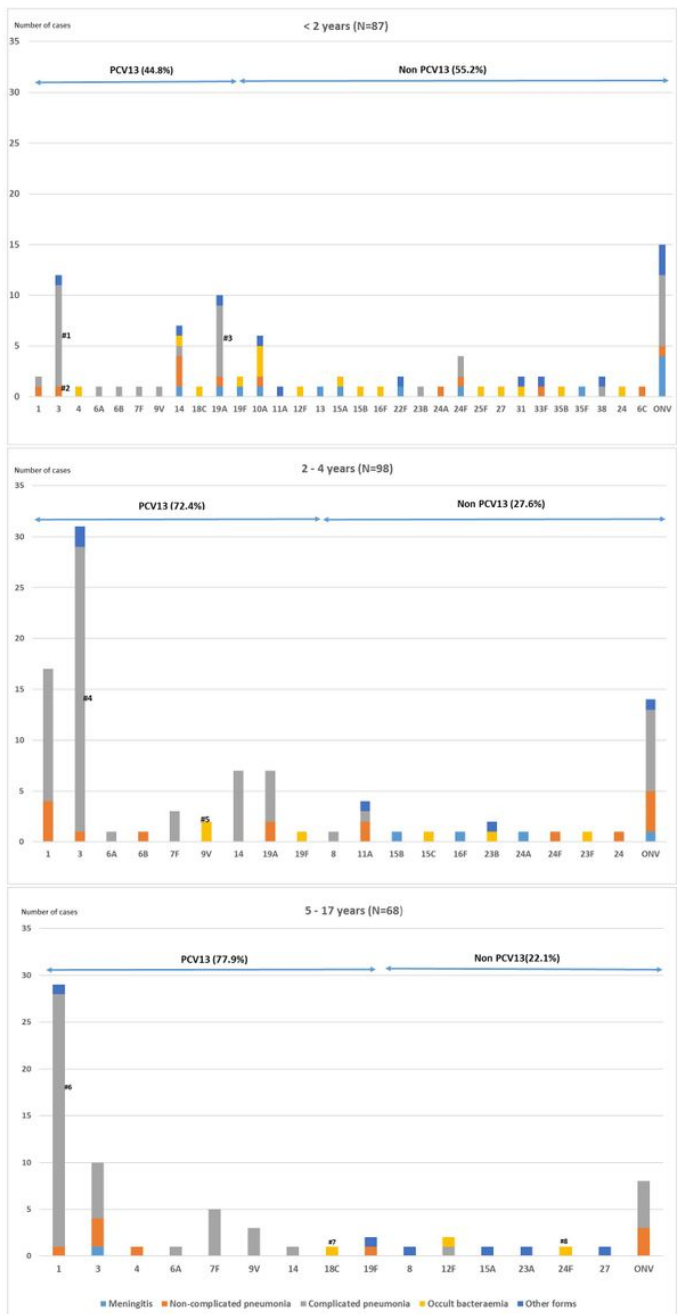

\section{Figure 3}

Distribution of Streptococcus pneumoniae serotypes in children $\leq 17$ years by clinical presentation. $\bullet<2$ age group. \#1: $p<0.001 ; \# 2$ : $p=0.004$ (Noncomplicated and complicated pneumonia); \#3: $p=0.033 . \cdot 2-4$ age group. \#4: $p=0.002 ; \# 5: p=0.003 \cdot 5-17$ age group. \#6:p<0.001; \#7: $p=0.004 ; \# 8$ : $p=0.004$ The $p$ value indicates differences in the relationship between a serotype and a clinical form with respect to the other serotypes. IPD: invasive pneumococcal disease. ONV: Other non-vaccine serotypes.

\section{Supplementary Files}

This is a list of supplementary files associated with this preprint. Click to download.

- IPDserotypeantimicrobialchildrenCatalonia20122016.xIsx 